\title{
Stackelberg Approach for Pricing Differentiated Services
}

\author{
Eitan Altman \\ INRIA, B.P. 93 \\ 2004 Route des Lucioles \\ 06902, Sophia-Antipolis \\ Cedex, France \\ altman@sophia.inria.fr
}

\author{
Richard Marquez \\ Cinvestav-IPN, Sección de \\ Mecatrónica \\ Electrical Engineering Dept. \\ Av. IPN 2508, Col. San Pedro \\ Zacatenco \\ C.P. 07360, Mexico City \\ rmarquez@cinvestav.mx
}

\author{
Rachid El-Azouzi \\ Universite d'Avignon et des \\ Pays de Vaucluse (IUP) \\ 339 chemin des Meinajariès, \\ B.P.1228 \\ 84911 Avignon Cedex 9, \\ France \\ rachid.elazouzi@univ- \\ avignon.fr
}

\author{
David Ros \\ GET/ENST Bretagne \\ Rue de la châtaigneraie CS \\ 17607 \\ 35567 Cesson Sevigne \\ Cedex, France \\ david.ros@enst- \\ bretagne.fr
}

\author{
Bruno Tuffin \\ IRISA/INRIA \\ Campus Universitaire de \\ Beaulieu \\ 35042 Rennes Cedex, France \\ btuffin@irisa.fr
}

\begin{abstract}
We consider in this paper a set of connections sharing a common bottleneck link. We assume first that we have data transfer using TCP congestion control protocol and realtime traffic using some TCP-friendly transport protocol that satisfies the same square-root formula for throughput. The performance measures are determined according to the operational parameters of a RED buffer management. Those parameters are assumed to be able to give differentiated services to the applications according to their choice of service class. In terms of loss probabilities and of throughputs, we consider a best effort type of service differentiation where the QoS of connections is not guaranteed, but by choosing a better (more expensive) service class, the QoS parameters of a session can be improved (as long as that session is the only one to change its service class). We assume however, that the system is dimensioned so as to satisfy some average delay requirement. The choice of a service class of an application will depend both on the utility as well as on the cost it has to pay. We first study the performance of each traffic source as a function of the connections' parameters and the pricing policy of the network. We then study the Stackelberg equilibrium, i.e. the service provider's problem of how to choose the pricing so as to maximize its utility, taking into account the reaction of the users.
\end{abstract}

Permission to make digital or hard copies of all or part of this work for personal or classroom use is granted without fee provided that copies are not made or distributed for profit or commercial advantage and that copies bear this notice and the full citation on the first page. To copy otherwise, to republish, to post on servers or to redistribute to lists, requires prior specific permission and/or a fee.

Valuetools '07, October 23-25, 2007, Nantes, France

Copyright 2007 ICST 978-963-9799-00-4.

\section{Keywords}

TCP, Buffer Management, RED/AQM, Stackelberg equilibrium, Pricing

\section{INTRODUCTION}

We study in this paper the performance of competing connections that share a bottleneck link. All connections are assumed to have controlled rates that are "TCP friendly", i.e. they satisfy the well known square-root relation between marking probability and throughput [10, 12, 13]. A RED buffer management is used for early drop of packets. We allow for service differentiation between the connections through the packet dropping (or marking) probability which may depend on the connection (or on the connection class). More specifically, we consider a buffer management scheme that uses a single averaged queue length to determine the dropping probabilities (similar to the way it is done in the RIO-C (coupled RIO) buffer management, see [14]); for any given averaged queue size, packets belonging to connections with higher priority have smaller probability of being dropped than those belonging to lower priority classes. We compute the throughput and the average drop probability for each connection. We assume in our model that the average queue size is a predetermined system parameter, i.e. there is a target value for the average queue length which implies also a fixed average queueing delay at the buffer. Since the throughputs of connections can vary (according to the price they pay), this means that the link rate is adapted to the connections' input rates so as to guarantee this desirable average queue length.

We then address the question of the choice of priorities. Given utilities that depend on the performance measures, on one hand, and on the cost for a given priority (i.e. the pricing strategy of the provider), on the other hand, each user is faced with an optimization problem, which we solve explicitly. This then allows us to determine the choice of pricing strategy by the service provider which maximizes its own 
profits; the solution to this bi-level optimization problem is known as the Stackelberg equilibrium. Note that throughout the paper, we use indifferently user or connection (we place ourselves at the transport/flow layer).

In a previous paper [3], we analyzed a related problem with TCP and CBR traffic sharing a common bottleneck buffer without a given target on the average queueing delay. This created a strong coupling between the different connections, so that the utility of a given connection was influenced by the user priority choices of all other connections. This gave rise to a more complicated modeling of the problem faced by the users (for a fixed pricing strategy of the provider) which was shown to be a non-cooperative game. Although some properties of the equilibrium of the game were obtained in [3], we were not able to obtain explicit formulae for the equilibrium priority choices and therefore did not treat the Stackelberg problem there.

Related references. We briefly mention other recent work in that area. Reference [6] has considered a related problem where the traffic generated by each session was modeled as a Poisson process, and the service time was exponentially distributed. The decision variables were the input rates and the performance measure was the goodput (output rates). The paper restricted itself to symmetric flows and symmetric equilibria and the pricing issue was not considered. In this framework, with a common RED buffer, it was shown that an equilibrium does not exist. An equilibrium was obtained and characterized for an alternative buffer management that was proposed, called VLRED. We note that, in contrast to [6], we do obtain an equilibrium when using RED. For other related papers, see for instance [11] (in which a priority game is considered for competing connections sharing a drop-tail buffer), [1] as well as the survey [2]. In [16], the authors present mechanisms (e.g., AIMD of TCP) to control end-user transmission rate into differentiated services Internet through potential functions and corresponding convergence to Nash equilibrium. These references have not studied the Stackelberg equilibrium concept.

Stackelberg equilibrium has been used in other contexts of networking in $[4,9]$. Both references consider $M / M / 1$ type models for congestion. In our paper we model both TCP behavior as well as real time traffic, both sharing a common RED type router as a bottleneck. Other Stackelberg games in networking which are not directly related to TCP or to RED are [7, 19].

The structure of this paper is as follows. In Section 2 we describe the model of RED, then in Section 3 we compute the throughputs and the loss probabilities of the connections for given priorities chosen by the connections. In Section 4 we obtain the optimal priority choices of the connections for given pricing strategies of the network. The optimal pricing is then discussed in Section 5.

\section{THE MODEL}

RED is based on the following idea: there are two thresholds $q_{\min }$ and $q_{\max }$ such that the drop probability is 0 if the average queue length $q$ is less than $q_{\min }, 1$ if it is above $q_{\max }$, and $p_{\max }\left(q-q_{\min }\right) /\left(q_{\max }-q_{\min }\right)$ if it is $q$ with $q_{\min }<$ $q<q_{\max }$; the latter is the congestion avoidance mode of operation. This is illustrated in Figure 1. $p_{\max }$ is the maximum loss probability in the congestion avoidance mode of operation.

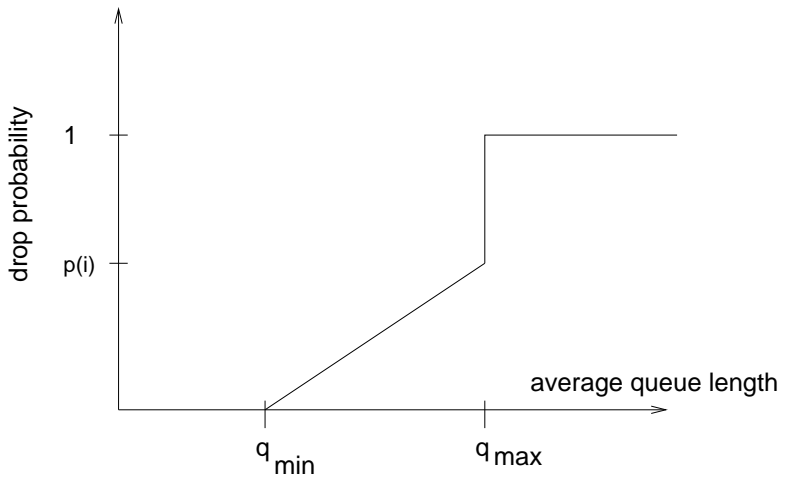

Figure 1: Drop probability in RED as function $q$

We consider a set $\mathcal{N}$ containing $N$ TCP flows (or TCP friendly flows) for data transfer and a set $\mathcal{I}$ containing $I$ real time flows, assumed to be TCP friendly (in the sense that they are rate controlled according so as to achieve the same throughput that TCP achieves as a function of loss probabilities and round trip time, see eq. (1)). We assume that connections can be differentiated by means of RED algorithm; they all share a common buffer yet RED treats them differently ${ }^{1}$. We assume that they all have common values of $q_{\min }$ and $q_{\max }$ but each flow $i$ may have a different value of $p_{\max }(i)$, which is the value of the drop probability as the average queue tends to $q_{\max }$ (from the left), see Figure 1. Denote $\mathbf{p}=\left\{p_{\max }(i), i \in \mathcal{I} \cup \mathcal{N}\right\}$. We identify $p_{\max }(i)$ as the priority class of a connection. The service rate of the bottleneck router is given by $\mu$.

\section{COMPUTING THE THROUGHPUTS}

We use the well-known relation for TCP rate which characterizes both data as well as real time connections (assumed to be TCP friendly):

$$
\lambda_{i}=\frac{1}{R_{i}} \sqrt{\frac{\theta}{p_{i}}}, \quad i \in \mathcal{N} \cup \mathcal{I},
$$

where $R_{i}$ and $p_{i}$ are TCP flow $i$ 's round trip time and drop probability, respectively. Parameter $\theta$ is typically taken as $3 / 2$ (when the delayed ack option is disabled) or $3 / 4$ (when it is enabled). We assume that the rates of connections are not limited by the receiver window.

We model the bottleneck as a fluid ${ }^{2}$ queue. We assume that the buffer size is well dimensioned, i.e. it is sufficiently large so that full utilization of the service rate can be achieved. This gives

$$
\sum_{j \in \mathcal{I} \cup \mathcal{N}} \lambda_{j}\left(1-p_{j}\right)=\mu .
$$

The parameters $\lambda_{j}$ and $p_{j}$ are determined from user $j$ choice of priority level. More precisely,

- $p_{j}$ 's are assumed to be controlled at the RED router; each $j, p_{j}$ is assumed to be a function of the priority

\footnotetext{
${ }^{1}$ RED punishes aggressive flows more by dropping more packets from those flows.

${ }^{2}$ In our study, the fluid (deterministic) assumption results in a simple yet suitable approximation, see [15].
} 
level of connection $j$ which is determined by user $j$ and which we identify with the price $x_{j}$ per packet payed by the $j$ th connection.

- If $\mu$ were fixed, the $p_{j}$ would then determine $\lambda_{j}$ through (1).

- We can thus view equation (2) as providing us with the rate $\mu$ that should be available at the bottleneck link so as to guarantee the performance measures (loss probabilities and throughputs) payed for by the connections.

Next, we define some pricing strategy that gives $p_{i}$ in terms of the flow rate $x_{i}$ of connection $i$. From Figure 1 for each $i$ the drop probability is

$$
p_{i}=p_{\max }(i) Q, \forall i, \quad \text { where } \quad Q=\frac{q-q_{\min }}{q_{\max }-q_{\min }} .
$$

We assume, as already mentioned in the introduction, that $q$ is a fixed target value of the average queue. Note that $0<Q<1$. We assume that the cost per packet $x_{i}$ is inversely proportional to $p_{\max }(i)$. Thus $p_{\max }(i)$ is given in terms of $x_{i}$ by $\left(\alpha x_{i}+\beta\right)^{-1}$ so that

$$
p_{i}\left(x_{i}\right)=\frac{Q}{\alpha x_{i}+\beta} .
$$

where fixed parameters $Q, \alpha, \beta$ stand for all connections. In addition, each connection may have a fixed subscription price $S$, independent of the quality of service.

For the network, the main difference between data connections and real-time connections can be represented in their different utilities, i.e. in the way they perceive quality of service and prices. Data connections using TCP are not sensitive to losses since lost packets are retransmitted allowing to recover packet losses. We thus assume that their utility has a component linear in the throughput and another one related to the costs. The connection utility for user $i(i \in \mathcal{N})$ is thus:

$$
U_{i}\left(x_{i}\right)=\lambda_{i}\left(x_{i}\right)\left(1-p_{i}\left(x_{i}\right)\right)-a_{i} \lambda_{i}\left(x_{i}\right)\left(1-p_{i}\left(x_{i}\right)\right) x_{i}-S
$$

where $a_{i}$ is a parameter representing the weight per unit flow rate of the price component in the utility. We consider a linear utility in this case to reflect that an increase in the throughput always affect significatively user welfare. It can be justified from a mathematical point of view, see $[3$, Footnote 2]. This also eases the analysis.

For real time connections, we assume that the utility has a component concave in the throughput, another component that represents (direct) sensitivity to losses and a component representing the evaluation of the cost for obtaining the requested priority level. We thus choose the utility function to be:

$$
\begin{aligned}
U_{i}\left(x_{i}\right)= & w_{i} \log _{10}\left(1+\lambda_{i}\left(x_{i}\right)\left(1-p_{i}\left(x_{i}\right)\right)\right) \\
& -b_{i} p_{i}\left(x_{i}\right)-a_{i} \lambda_{i}\left(x_{i}\right)\left(1-p_{i}\left(x_{i}\right)\right) x_{i}-S
\end{aligned}
$$

with $w_{i}$ and $b_{i}$ constant values depending on each connection $i$. As usual, $w_{i}>0$ corresponds to a preference parameter. The logarithmic function ${ }^{3}$ indicates a quality feature of real time connections: user reward do not increase in high flow-rate connections as fast as for slow flow-rate connections (consider for instance a voice or video connection,

${ }^{3}$ It is very common to use concave payoff functions for user utilities, see [4]. after some point the user do not detect significant changes when increasing quality service). Notice the fixed average queueing delay has not been directly considered in the above selected utility functions.

\section{OPTIMIZING USER'S PRICES}

\subsection{A numerical example}

We begin with a numerical example. The network parameters are given by $Q=0.5, \alpha=10, \beta=200$; the parameters describing data or real time connections are $\forall i R_{i}=0.05$, $\theta=1.5, a_{i}=0.01$. (We do not specify the value of $S$ since it is a constant that does not affect the optimization problem faced by a user.) Furthermore the real time connections are assumed to be such that $b_{i}=0.1$ and $w_{i}=1600 \forall i$. The functions (5) and (6) are depicted in Figures 2-3 as a function of the price $x$ (equivalent to giving the priority level). Their restriction to nonnegative $x$ provides the utilities. We

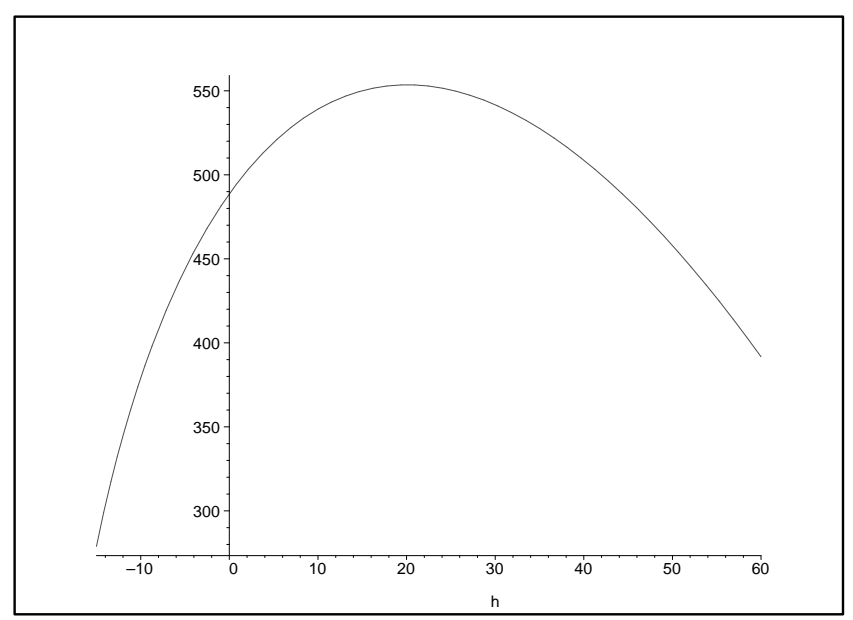

Figure 2: Utility as a function of the priority for data traffic

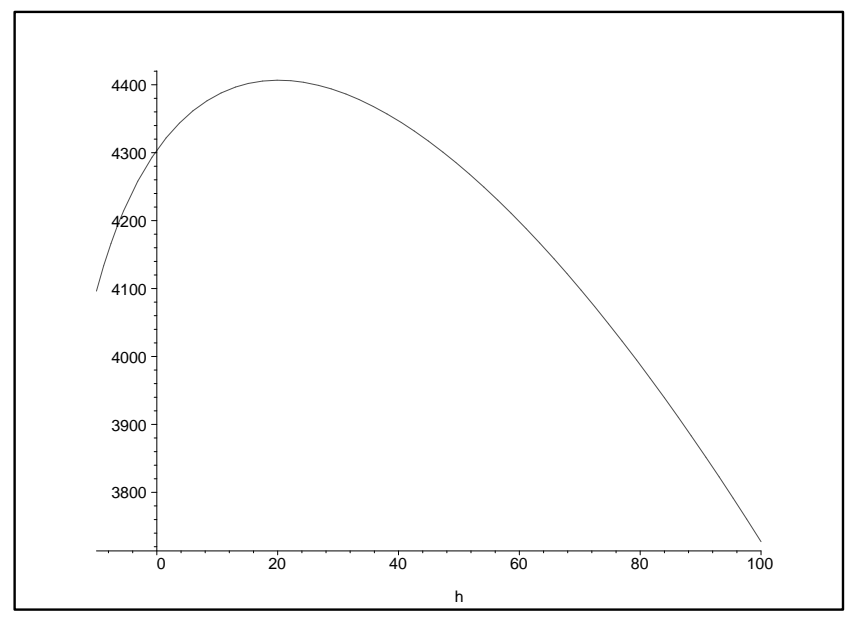

Figure 3: Utility as a function of the priority for real time traffic

see that the utility as a function of the priority level $x$ has a clear unique maximum. We shall prove this observation 
in the next subsection for general choices of parameters for data connections.

Below we address the question of how user $i$ should select a priority level $x$ so that the resulting parameters $\lambda_{i}(x)$ and $p_{i}(x)$ maximize its utility. This will determine the reaction of users to a given pricing strategy of the network. In the next section we then introduce the network's utility which will be used to compute the network's pricing strategy that maximizes its utility when taking into account users reaction to that strategy.

\subsection{Analysis of the first level optimization prob- lem}

\section{Data connections.}

We will consider first the connection utility given by (5).

Proposition 1. There exists a unique maximum of $U_{i}\left(x_{i}\right)$ at $X_{i}>0$ for data connections. If

$$
a_{i}<\frac{\alpha}{2 \beta} \frac{\beta+Q}{\beta-Q},
$$

it is the unique solution of

$$
X_{i}=\left\{x:\left.\frac{d U_{i}\left(x_{i}\right)}{d x_{i}}\right|_{x}=0\right\}
$$

i.e.,

$$
\begin{aligned}
X_{i}=\frac{1}{\alpha 6} & \left(\frac{\alpha}{a_{i}}-5 \beta+Q\right. \\
& +\sqrt{\left.\left(\frac{\alpha}{a_{i}}+\beta+Q\right)^{2}+12 Q\left(\frac{\alpha}{a_{i}}+\beta\right)\right)}
\end{aligned}
$$

Otherwise the maximum is located at $X_{i}=0$.

Proof: The partial derivatives of $U_{i}\left(x_{i}\right), \frac{d U_{i}}{d x_{i}}\left(x_{i}\right)$, are proportional to

$$
\begin{aligned}
H\left(X_{i}, a_{i}\right)= & {\left[\sqrt{\alpha x_{i}+\beta}+\frac{Q}{\sqrt{\alpha x_{i}+\beta}}\right] \times } \\
& \left(\frac{\alpha\left(1-a_{i} x_{i}\right)}{2\left(\alpha x_{i}+\beta\right)}-a_{i}\right)+\frac{2 a_{i} Q}{\sqrt{\alpha x_{i}+\beta}} .
\end{aligned}
$$

The unique solution $X_{i}$ to $\left.\frac{d U_{i}}{d x_{i}}\left(x_{i}\right)\right|_{X_{i}}=H\left(X_{i}, a_{i}\right)=0$ is given by (7).

We have $\lim _{x_{i} \rightarrow+\infty} \frac{d U_{i}}{d x_{i}}\left(x_{i}\right) \rightarrow-\infty$; straightforward calculations show that $\frac{d U_{i}}{d x_{i}}(0)>0$ if and only if $a_{i}<\frac{\alpha}{2 \beta} \frac{\beta+Q}{\beta-Q}$, thus the solution $X_{i}$ corresponds to an absolute maximum of the function $U_{i}\left(x_{i}\right)$ [18]. Otherwise, if $\frac{d U_{i}}{d x_{i}}(0) \leq 0$ we have an absolute maximum at $X_{i}=0$ because the function $U_{i}\left(x_{i}\right)$ is strictly decreasing for all $x_{i} \geq 0$.

Remark that, using this proposition, the drop probability $p_{i}\left(X_{i}\right)$ is given by

$$
p_{i}\left(X_{i}\right)=\frac{6 Q}{\left(\varphi_{i}+Q+\sqrt{\left(\varphi_{i}+Q\right)^{2}+12 Q \varphi_{i}}\right)}
$$

if $a_{i}<\frac{\alpha}{2 \beta} \frac{\beta+Q}{\beta-Q}$, where $\varphi_{i}=\frac{\alpha}{a_{i}}+\beta$, and $p(0)=Q / \beta$ otherwise.

\section{Real-time connections.}

Similarly to the approach for the data connections, one can numerically get the optimal choice $x_{i}$ for real-time connections which maximize user utilities $U_{i}\left(x_{i}\right)$. We have not been able to determine sufficient conditions for uniqueness for real-time connections in full generality; we leave it for future work. Nevertheless, the uniqueness can be observed for instance in Figure 3.

From now, we focus only on data users. To illustrate Proposition 1 we consider the following example. the parameter values $Q=0.5, \alpha=10, \beta=20, \theta=1, R_{i}=0.05$, $a_{i}=0.1 \forall i$. The value of $\frac{\alpha}{2 \beta} \frac{\beta+Q}{\beta-Q}$ results in $0.2628>0.1=$ $a_{i}$. Figure 4 shows the utility $U_{i}(x)$ of a single data connection as a function of $x$ for these parameters. The maximum is then obtained at $X_{i}=2.06>0$ as expected.

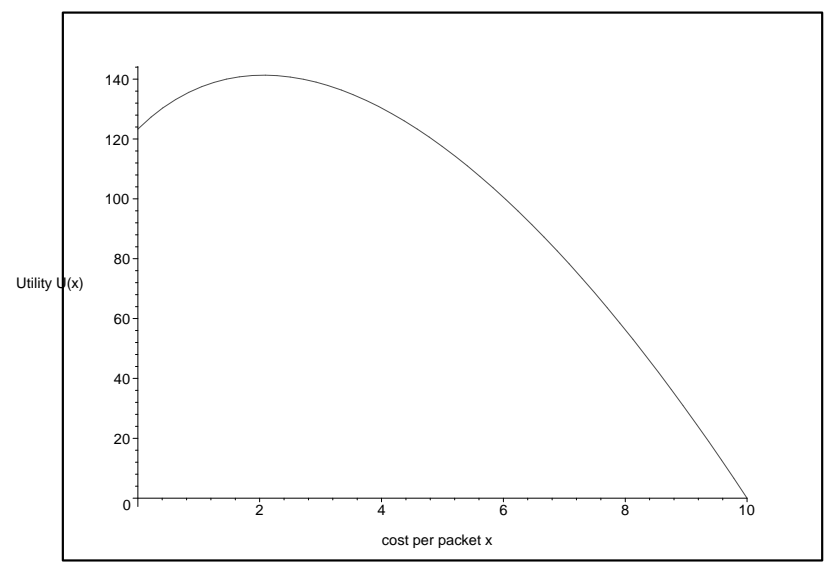

Figure 4: Utility as a function of $x$

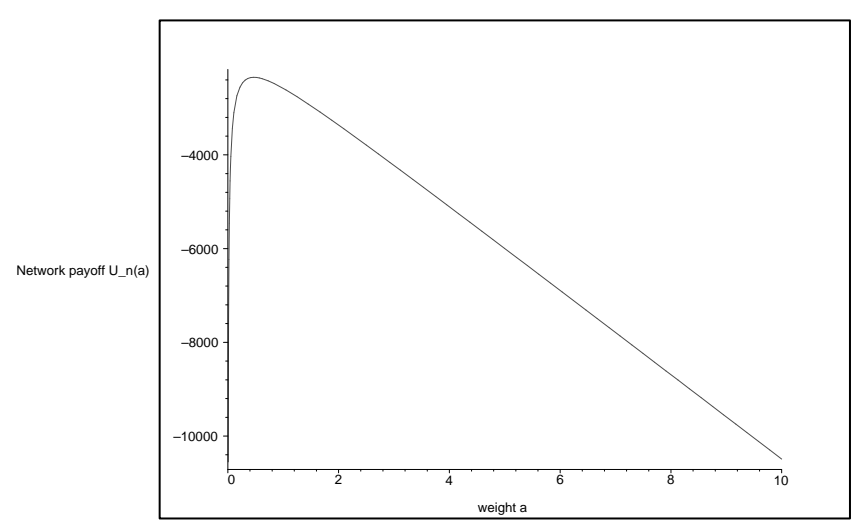

Figure 5: Network payoff as a function of $a$

\section{OPTIMIZING THE NETWORK'S PRIC- ING STRATEGY}

We assume that the network's payoff is made of two components: a cost which is proportional to the resources it provides, that is to the link rate $\mu$, and a utility that represents its revenues. The latter is assumed to be simply the sum of prices payed by the users. Considering a Stackelberg 
approach, the network's utility is thus given by:

$$
\begin{aligned}
\mathcal{U}_{\text {network }}\left(a_{i}\right)= & \sum_{i \in \mathcal{I} \cup \mathcal{N}}\left(a_{i} \lambda_{i}\left(X_{i}\right)\left(1-p_{i}\left(X_{i}\right)\right) X_{i}+S\right)-\delta \mu \\
= & \sum_{i \in \mathcal{I} \cup \mathcal{N}}\left(a_{i} \lambda_{i}\left(X_{i}\right)\left(1-p_{i}\left(X_{i}\right)\right) X_{i}+S\right) \\
& -\delta \sum_{i \in \mathcal{I} \cup \mathcal{N}} \lambda_{i}\left(X_{i}\right)\left(1-p_{i}\left(X_{i}\right)\right)
\end{aligned}
$$

i.e., it depends on the equilibrium obtained at users' level.

\section{Identical data connections.}

Consider $N$ identical data users, i.e., $X_{i}=X=X(a)>0$, given by (7), $a_{i}=a, \lambda_{i}=\lambda$, and $R_{i}=R$. Network utility $\mathcal{U}_{\text {network }}$ simplifies to

$\mathcal{U}_{\text {network }}(a)=N(a X(a)-\delta) \lambda(X(a))(1-p(X(a)))+N \cdot S$.

As before, the maximum of (9) is obtained by using equation $d \mathcal{U}_{\text {network }}(a) / d a=0$. The solution to this expression depends also on $\delta$. In particular, for $\delta=1, \mathcal{U}_{\text {network }}(a)$ has a maximum $a=\frac{\alpha}{2 \beta} \frac{\beta+Q}{\beta-Q}>0$. For $\delta>1$ then it yields $a>\frac{\alpha}{2 \beta} \frac{\beta+Q}{\beta-Q}$. In these two cases, i.e., when the optimal parameter $a$, that the network imposes, satisfies $a \geq \frac{\alpha}{2 \beta} \frac{\beta+Q}{\beta-Q}$, the utility of the users has an absolute maximum at $X=0$, see Proposition 1 . This means that they subscribe to the minimal quality of service: they pay only the subscription fee $S$ and obtain the largest drop probability, $Q / \beta$ (and hence the minimal throughput). Consider a numerical example, illustrated on Figure 5: a plot of $U(a)$ is given for $N=10$ identical data connections, for $\delta=2$, $Q=20 / 40=0.5, \alpha=10, \beta=20, c=1, R=0.05$. The maximum then is obtained at $a=0.4708188006>0.2628$.

At $0<D<\delta<1$, it yields a reasonable value $0<$ $a<\frac{\alpha}{2 \beta} \frac{\beta+Q}{\beta-Q}$; the quantity $D$ is such that the absolute maximum is obtained at $a=0$, on the interval $[0, \infty)$. In Figure $6 \mathrm{a}$ we present a plot for $\delta=35 / 100$, where $a=$ $0.01255526875<0.2628$. We have $D=0.3333$. In this case we obtain $X=25.28249042$, the cost per packet for the maximum user's utility, see Figure $6 \mathrm{~b}$.

\section{CONCLUSIONS}

In this paper, we have analyzed a Stackelberg game where users choose the price they are willing to pay at a RED buffer in order to discriminate service. With respect to a previous result, we use the fact that there is a fixed target for the average queue length (thus resulting also a fixed average queueing delay). This simplification allows to obtain explicit formulae for the equilibrium priority choices of users. Thanks to those expressions, we are able to solve the Stackelberg formulation of the problem.

\section{ACKNOWLEDGMENTS}

This work has been supported in part by an ECOS-NORD grant PI-2003000207/V04M01. Fruitful discussions with Dr. Tania Jiménez are gratefully acknowledged.

\section{REFERENCES}

a)
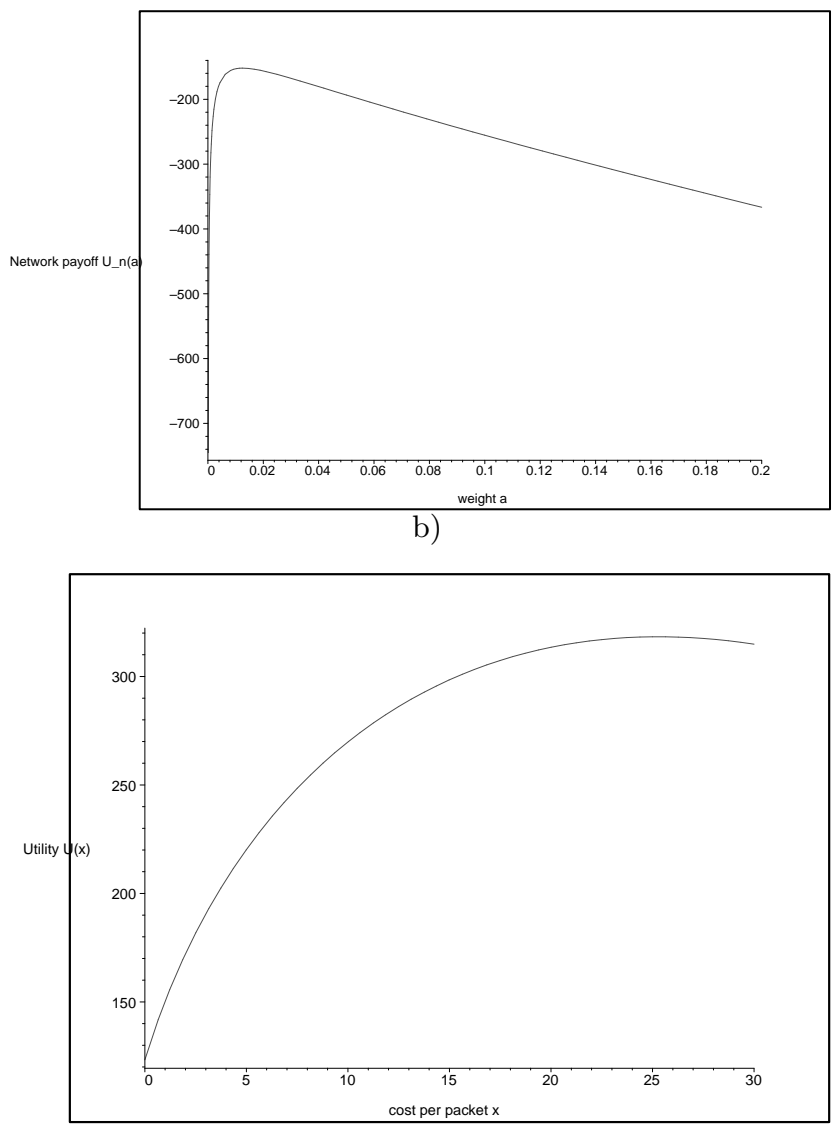

Figure 6: Network payoff as a function of $a$; case $a<\frac{\alpha}{2 \beta} \frac{\beta+Q}{\beta-Q}$

[1] T. Alpcan and T. Basar, "A game-theoretic framework for congestion control in a general topology networks", 41st IEEE Conference on Decision and Control, Las Vegas, Nevada, Dec. 10-13, 2002.

[2] E. Altman, T. Boulogne, R. El Azouzi, T. Jimenez and L. Wynter, "A survey on networking games", Computers and Operations Research, Vol. 33, Issue 2, 286-311, 2006.

[3] E. Altman, R. El-Azouzi, D. Barman, D. Ross and B. Tuffin, "Pricing Differential Services: A Game-Theoretic Approach", Computer Networks, 50(7), pp. 982-1002, 2006.

[4] T. Basar and R. Srikant, "A Stackelberg network game with a large number of followers", J. Optimization Theory and Applications, 115(3):479-490, December 2002

[5] F. Bernstein and A. Federgruen, "A general equilibrium model for decentralized supply chains with price- and service-competition", Available at http://faculty.fuqua.duke.edu/ fernando/bio/

[6] D. Dutta, A. Goel and J. Heidemann, "Oblivious AQM and Nash Equilibria", IEEE Infocom, 2003.

[7] Rahul Garg, Abhinav Kamra, Varun Khurana, "A game-theoretic approach towards congestion control in 
communication networks", ACM SIGCOMM

Computer Communication Review, Volume 32 Issue 3, July 2002.

[8] J. Janssen, D. De Vleeschauwer, M. Büchli and G. H. Petit, "Assessing voice quality in packet-based telephony", IEEE Internet Computing, pp. 48-56, May-June, 2002.

[9] Y. A. Korilis, A. A. Lazar and A. Orda, "Achieving network optima using Stackelberg routing strategies", IEEE/ACM Transactions on Networking, 5(1), pp. 161-173, 1997.

[10] T.V. Lakshman and U. Madhow, "The performance of TCP/IP for networks with high bandwidth-delay products and random loss", IEEE/ACM Transactions on Networking, Jun 1997.

[11] M. Mandjes, "Pricing strategies under heterogeneous service requirements", Computer Networks 42, pp. 231-249, 2003.

[12] M. Mathis, J. Semke, J. Mahdavi, and T. Ott, "The Macroscopic Behavior of the TCP Congestion Avoidance Algorithm", ACM Computer Communication Review, Jul 1997.

[13] V. Mistra, W.-B. Gong, and D. Towsley, "Stochastic differential equation modeling and analysis of TCP-windowsize behaviour", Performance, Oct 1999.

[14] P. Pieda, J. Ethridge, M. Baines and F. Shallwani, A Network Simulator Differentiated Services Implementation, Open IP, Nortel Networks, July, 2000. Available at http://www.isi.edu/nsnam/ns

[15] S. Shakkottai, R. Srikant, "How good are deterministic fluid models of Internet congestion control", IEEE INFOCOM '02

[16] Y. Jin, G. Kesidis, "Nash equilibria of a generic networking game with applications to circuit-switched networks", IEEE INFOCOM '03

[17] Numerical Recipes in C, The Art of Scientific Computing, 2nd Edition, Section 5.6 http://www.ulib.org/webRoot/Books/Numerical_ Recipes/bookc.html1

[18] W. Rudin, Functional Analysis, McGraw-Hill, New York, 1973

[19] S. Shakkottai, R. Srikant, "Economics of network pricing with multiple ISPs", IEEE/ACM Transactions on Networking (TON), Volume 14 Issue 6, December 2006 . 